KYUNGPOOK Math. J. 54(2014), 677-684

http://dx.doi.org/10.5666/KMJ.2014.54.4.677

\title{
Generalization of a Transformation Formula for the Exton's Triple Hypergeometric Series $X_{12}$ and $X_{17}$
}

\author{
JUNESANG CHOI* \\ Department of Mathematics, Dongguk University, Gyeongju 780-714, Republic of \\ Korea \\ e-mail : junesang@mail.dongguk.ac.kr \\ ArJun K. Rathie \\ Department of Mathematics, School of Mathematical \& Physical Sciences, Cen- \\ tral University of Kerala, Riverside Transit Campus, Padennakad P.O. Nileshwar, \\ Kasaragod-671 328, India \\ e-mail : akrathie@gmail.com
}

ABSTRACT. In the theory of hypergeometric functions of one or several variables, a remarkable amount of mathematicians's concern has been given to develop their transformation formulas and summation identities. Here we aim at generalizing the following transformation formula for the Exton's triple hypergeometric series $X_{12}$ and $X_{17}$ :

$$
\begin{gathered}
(1+2 z)^{-b} X_{17}\left(a, b, c_{3} ; c_{1}, c_{2}, 2 c_{3} ; x, \frac{y}{1+2 z}, \frac{4 z}{1+2 z}\right) \\
=X_{12}\left(a, b ; c_{1}, c_{2}, c_{3}+\frac{1}{2} ; x, y, z^{2}\right) .
\end{gathered}
$$

The results are derived with the help of two general hypergeometric identities for the terminating ${ }_{2} F_{1}(2)$ series which were very recently obtained by Kim et al. Four interesting results closely related to the Exton's transformation formula are also chosen, among ten, to be derived as special illustrative cases of our main findings. The results easily obtained in this paper are simple and (potentially) useful.

\section{Introduction and Preliminaries}

In 1982, Exton [6] published a very interesting and useful research paper in

* Corresponding Author.

Received November 17, 2012; accepted March 27, 2013.

2010 Mathematics Subject Classification: Primary 33C70, 33C065; Secondary 33C90, $33 \mathrm{C} 0$.

Key words and phrases: Hypergeometric functions of several variables, Multiple Gaussian hypergeometric series, Exton's triple hypergeometric series, Gauss's hypergeometric functions. 
which he encountered a number of triple hypergeometric functions of second order whose series representations involve such products as $(a)_{2 m+2 n+p}$ and $(a)_{2 m+n+p}$ and introduced a set of 20 distinct triple hypergeometric functions $X_{1}$ to $X_{20}$ and also gave their integral representations of Laplacian type which include the confluent hypergeometric functions ${ }_{0} F_{1},{ }_{1} F_{1}$, a Humbert function $\psi_{1}$ and a Humbert function $\phi_{2}$ in their kernels. It is not out of place to mention here that the Exton's functions $X_{1}$ to $X_{20}$ have been studied a lot until today, see, for example, the works [2], [3], [4], [5], [8], [9], [10] and [11]. Moreover, Exton [6] presented a large number of very interesting transformation formulas and reducible cases with the help of two known results which are called in the literature as Kummer's first and second transformations or theorems.

Here we are interested in the following Exton's triple hypergeometric series $X_{12}$ defined by

$$
X_{12}\left(a, b ; c_{1}, c_{2}, c_{3} ; x, y, z\right)=\sum_{m=0}^{\infty} \sum_{n=0}^{\infty} \sum_{r=0}^{\infty} \frac{(a)_{2 m+n}(b)_{n+2 r}}{\left(c_{1}\right)_{m}\left(c_{2}\right)_{n}\left(c_{3}\right)_{r}} \frac{x^{m}}{m !} \frac{y^{n}}{n !} \frac{z^{r}}{r !}
$$

and its integral representation is given by

$$
\begin{aligned}
& X_{12}\left(a, b ; c_{1}, c_{2}, c_{3} ; x, y, z\right)=\frac{1}{\Gamma(a) \Gamma(b)} \\
& \cdot \int_{0}^{\infty} \int_{0}^{\infty} e^{-s-t} s^{a-1} t^{b-1}{ }_{0} F_{1}\left[\begin{array}{l}
-; \\
c_{1} ;
\end{array} s^{2}\right]{ }_{0} F_{1}\left[\begin{array}{l}
-; \\
c_{2} ;
\end{array} y t\right]{ }_{0} F_{1}\left[\begin{array}{l}
-; \\
c_{3} ;
\end{array} t^{2}\right] d s d t
\end{aligned}
$$

and Exton's triple hypergeometric series $X_{17}$ defined by

$$
X_{17}\left(a, b, c ; d_{1}, d_{2}, d_{3} ; x, y, z\right)=\sum_{m=0}^{\infty} \sum_{n=0}^{\infty} \sum_{r=0}^{\infty} \frac{(a)_{2 m+n}(b)_{n+r}(c)_{r}}{\left(d_{1}\right)_{m}\left(d_{2}\right)_{n}\left(d_{3}\right)_{r}} \frac{x^{m}}{m !} \frac{y^{n}}{n !} \frac{z^{r}}{r !}
$$

and its integral representation is given by

$$
\begin{aligned}
& X_{17}\left(a, b, c ; d_{1}, d_{2}, d_{3} ; x, y, z\right)=\frac{1}{\Gamma(a) \Gamma(c)} \\
& \cdot \int_{0}^{\infty} \int_{0}^{\infty} e^{-s-t} s^{a-1} t^{c-1}{ }_{0} F_{1}\left[\begin{array}{c}
-; \\
d_{1} ;
\end{array} s^{2}\right] \psi_{2}\left(b ; d_{2}, d_{3} ; y s, z t\right) d s d t
\end{aligned}
$$

where $\psi_{2}$ denotes a Humbert function (see, for example, [13, p. 25]).

In the above definitions and their integral representations, the generalized hypergeometric series ${ }_{p} F_{q}$ is defined by (see $[12$, p. 73$]$ ):

$$
\begin{aligned}
{ }_{p} F_{q} & {\left[\begin{array}{c}
\alpha_{1}, \ldots, \alpha_{p} ; \\
\beta_{1}, \ldots, \beta_{q} ;
\end{array}\right]=\sum_{n=0}^{\infty} \frac{\left(\alpha_{1}\right)_{n} \cdots\left(\alpha_{p}\right)_{n}}{\left(\beta_{1}\right)_{n} \cdots\left(\beta_{q}\right)_{n}} \frac{z^{n}}{n !} } \\
& ={ }_{p} F_{q}\left(\alpha_{1}, \ldots, \alpha_{p} ; \beta_{1}, \ldots, \beta_{q} ; z\right) \quad\left(p, q \in \mathbb{N}_{0}\right),
\end{aligned}
$$


where $(\lambda)_{\nu}$ denotes the Pochhammer symbol or the shifted factorial, since

$$
(1)_{n}=n ! \quad\left(n \in \mathbb{N}_{0}:=\mathbb{N} \cup\{0\} ; \mathbb{N}:=\{1,2,3, \cdots\}\right),
$$

which is defined (for $\lambda, \nu \in \mathbb{C}$ ), in terms of the familiar Gamma function $\Gamma$, by

$$
=\left\{\begin{array}{lr}
1 & (\nu=0 ; \lambda \in \mathbb{C} \backslash\{0\}) \\
\lambda(\lambda+1) \cdots(\lambda+n-1) & (\nu=n \in \mathbb{N} ; \lambda \in \mathbb{C}),
\end{array}\right.
$$

it being understood conventionally that $(0)_{0}:=1$ and $\mathbb{C}$ the set of complex numbers.

The precise three-dimensional region of convergence of (1.1) and (1.3) can be seen in the monograph of Srivastava and Karlsson [13, pp. 87-107].

In the theory of hypergeometric functions of one or several variables, a remarkable amount of mathematicians's concern has been given to develop their transformation formulas and summation identities. Here we aim at generalizing the following transformation formula for the Exton's triple hypergeometric series $X_{12}$ and $X_{17}$ :

$$
\begin{gathered}
(1+2 z)^{-b} X_{17}\left(a, b, c_{3} ; c_{1}, c_{2}, 2 c_{3} ; x, \frac{y}{1+2 z}, \frac{4 z}{1+2 z}\right) \\
=X_{12}\left(a, b ; c_{1}, c_{2}, c_{3}+\frac{1}{2} ; x, y, z^{2}\right) .
\end{gathered}
$$

It is noted that, for convenience and simplicity, we will use

$$
\sum_{m, n, r=0}^{\infty} \text { for } \sum_{m=0}^{\infty} \sum_{n=0}^{\infty} \sum_{r=0}^{\infty} \text {. }
$$

In order to obtain a natural generalization of the Exton's transformation formula (1.7), we require the following results recently obtained by Kim et al. [7]:

$$
\begin{aligned}
{ }_{2} F_{1} & {\left[\begin{array}{c}
-2 n, \alpha ; \\
2 \alpha+j ;
\end{array}\right] } \\
& =\mathcal{A}_{j} \frac{\Gamma(\alpha) \Gamma(1-\alpha)\left(\frac{1}{2}\right)_{n}\left(\alpha+\left[\frac{j+1}{2}\right]\right)_{n}}{\Gamma\left(\alpha+\frac{1}{2} j+\frac{1}{2}|j|\right) \Gamma\left(1-\alpha-\left[\frac{j+1}{2}\right]\right)\left(\alpha+\frac{1}{2} j\right)_{n}\left(\alpha+\frac{1}{2} j+\frac{1}{2}\right)_{n}}
\end{aligned}
$$

and

$$
\begin{aligned}
{ }_{2} F_{1} & {\left[\begin{array}{r}
-2 n-1, \alpha ; \\
2 \alpha+j ;
\end{array}\right] } \\
& =\frac{\mathcal{B}_{j}}{2 \alpha+j} \frac{\Gamma(-\alpha) \Gamma(\alpha+1)\left(\frac{3}{2}\right)_{n}\left(1+\alpha+\left[\frac{j}{2}\right]\right)_{n}}{\Gamma\left(\alpha+\frac{1}{2} j+\frac{1}{2}|j|\right) \Gamma\left(-\alpha-\left[\frac{j}{2}\right]\right)\left(\alpha+\frac{1}{2} j+\frac{1}{2}\right)_{n}\left(\alpha+\frac{1}{2} j+1\right)_{n}},
\end{aligned}
$$


where $n \in \mathbb{N}_{0}, j=0, \pm 1, \ldots, \pm 5,[x]$ is the greatest integer less than or equal to $x$ and its modulus is denoted by $|x|$, and the coefficients $\mathcal{A}_{j}$ and $\mathcal{B}_{j}$ are given in the following table.

TABLE

\begin{tabular}{|r|c|c|}
\hline$j$ & $\mathcal{A}_{j}$ & $\mathcal{B}_{j}$ \\
\hline \hline 5 & $-4(1-\alpha-2 n)^{2}+2(1-\alpha)(1-\alpha-2 n)$ & $4(\alpha+2 n)^{2}-2(1-\alpha)(\alpha+2 n)$ \\
& $+(1-\alpha)^{2}+22(1-\alpha-2 n)$ & $+(1-\alpha)^{2}+34(\alpha+2 n)$ \\
& $-13(1-\alpha)-20$ & $+(1-\alpha)+62$ \\
\hline 4 & $2(\alpha+1+2 n)(\alpha+3+2 n)-\alpha(\alpha+3)$ & $4(\alpha+2 n+3)$ \\
\hline 3 & $-\alpha-4 n-2$ & $-3 \alpha-4 n-6$ \\
\hline 2 & $-\alpha-1-2 n$ & -2 \\
\hline 1 & -1 & 1 \\
\hline 0 & 1 & 2 \\
\hline-1 & 1 & $3-3 \alpha-4 n$ \\
\hline-2 & $1-\alpha-4 n$ & $4(1-\alpha-2 n)$ \\
\hline-3 & $-(1-\alpha)^{2}+8(1-\alpha-2 n)+7 \alpha-7$ & $-(1-\alpha)^{2}-16(\alpha+2 n)+\alpha-1$ \\
\hline-4 & $2(1-2 \alpha-n)(3-\alpha-2 n)-(1-\alpha)(4-\alpha)$ & $4(\alpha+2 n)^{2}+2(1-\alpha)(\alpha+2 n)$ \\
\hline-5 & $4(1-\alpha-2 n)^{2}-2(1-\alpha)(1-\alpha-2 n)$ & \\
& &
\end{tabular}

\section{Main Transformation Formulas}

A natural generalization of Exton's transformation formula (1.7) to be established is as follows:

$$
\begin{aligned}
& (1+2 z)^{-b} X_{17}\left(a, b, c_{3} ; c_{1}, c_{2}, 2 c_{3}+j ; x, \frac{y}{1+2 z}, \frac{4 z}{1+2 z}\right) \\
& =\frac{\Gamma\left(c_{3}\right) \Gamma\left(1-c_{3}\right)}{\Gamma\left(c_{3}+\frac{1}{2} j+\frac{1}{2}|j|\right) \Gamma\left(1-c_{3}-\left[\frac{j+1}{2}\right]\right)} \\
& \quad \cdot \sum_{m, n, r=0}^{\infty} \mathcal{C}_{j} \frac{(a)_{2 m+n}(b)_{n+2 r}\left(c_{3}+\left[\frac{j+1}{2}\right]\right)_{r}}{\left(c_{1}\right)_{m}\left(c_{2}\right)_{n}\left(c_{3}+\frac{1}{2} j\right)_{r}\left(c_{3}+\frac{1}{2} j+\frac{1}{2}\right)_{r}} \frac{x^{m}}{m !} \frac{y^{n}}{n !} \frac{z^{2 r}}{r !} \\
& -\frac{2 b z}{2 c_{3}+j} \frac{\Gamma\left(-c_{3}\right) \Gamma\left(1+c_{3}\right)}{\Gamma\left(c_{3}+\frac{1}{2} j+\frac{1}{2}|j|\right) \Gamma\left(-c_{3}-\left[\frac{j}{2}\right]\right)} \\
& \cdot \sum_{m, n, r=0}^{\infty} \mathcal{D}_{j} \frac{(a)_{2 m+n}(b+1)_{n+2 r}\left(1+c_{3}+\left[\frac{j}{2}\right]\right)_{r}}{\left(c_{1}\right)_{m}\left(c_{2}\right)_{n}\left(c_{3}+\frac{1}{2} j+\frac{1}{2}\right)_{r}\left(c_{3}+\frac{1}{2} j+1\right)_{r}} \frac{x^{m}}{m !} \frac{y^{n}}{n !} \frac{z^{2 r}}{r !} \\
& \quad(j=0, \pm 1, \ldots, \pm 5),
\end{aligned}
$$

where the coefficients $\mathcal{C}_{j}$ and $\mathcal{D}_{j}$ here can be obtained by simply changing $n$ and $\alpha$ into $r$ and $c_{3}$, respectively, in the Table of $\mathcal{A}_{j}$ and $\mathcal{B}_{j}$. 
Proof. For convenience and simplicity, by denoting the left-hand side of (2.1) by $S$ and using the series definition of $X_{17}$ as given in (1.3), after a little simplification, we have

$$
S=\sum_{m, n, p=0}^{\infty} \frac{(a)_{2 m+n}(b)_{n+p}\left(c_{3}\right)_{p}}{\left(c_{1}\right)_{m}\left(c_{2}\right)_{n}\left(2 c_{3}+j\right)_{p}} \frac{x^{m}}{m !} \frac{y^{n}}{n !} \frac{z^{p}}{p !} 2^{2 p}(1+2 z)^{-(b+n+p)} .
$$

Using the binomial theorem (see [12, p. 58]) for the last factor, we get

$$
S=\sum_{m, n, p, r=0}^{\infty} \frac{(a)_{2 m+n}(b)_{n+p}\left(c_{3}\right)_{p}}{\left(c_{1}\right)_{m}\left(c_{2}\right)_{n}\left(2 c_{3}+j\right)_{p}} \frac{x^{m}}{m !} \frac{y^{n}}{n !} \frac{z^{p}}{p !} 2^{2 p} \frac{(b+n+p)_{r}}{r !}(-1)^{r} 2^{r} z^{r} .
$$

Using the identity $(b)_{n+p}(b+n+p)_{r}=(b)_{n+p+r}$, after a little simplification, we obtain

$$
S=\sum_{m, n, p, r=0}^{\infty} \frac{(a)_{2 m+n}(b)_{n+p+r}\left(c_{3}\right)_{p}}{\left(c_{1}\right)_{m}\left(c_{2}\right)_{n}\left(2 c_{3}+j\right)_{p}}(-1)^{r} 2^{2 p+r} \frac{x^{m}}{m !} \frac{y^{n}}{n !} \frac{z^{p+r}}{p ! r !}
$$

Now using the following well known formal manipulation of double series (see [12, p. 56]; for other manipulations, see also [1, Eq. (1.4)]):

$$
\sum_{r=0}^{\infty} \sum_{p=0}^{\infty} A(p, r)=\sum_{r=0}^{\infty} \sum_{p=0}^{r} A(p, r-p),
$$

after a little simplification, we have

$$
S=\sum_{m, n, r=0}^{\infty} \sum_{p=0}^{r} \frac{(a)_{2 m+n}(b)_{n+r}\left(c_{3}\right)_{p}}{\left(c_{1}\right)_{m}\left(c_{2}\right)_{n}\left(2 c_{3}+j\right)_{p}}(-1)^{r-p} 2^{p+r} \frac{x^{m}}{m !} \frac{y^{n}}{n !} \frac{z^{r}}{p !(r-p) !} .
$$

Using the following formula

$$
(r-p) !=\frac{(-1)^{p} r !}{(-r)_{p}} \quad\left(0 \leqq p \leqq r ; r, p \in \mathbb{N}_{0}\right)
$$

after a little simplification, we get

$$
S=\sum_{m, n, r=0}^{\infty} \frac{(a)_{2 m+n}(b)_{n+r}(-2)^{r}}{\left(c_{1}\right)_{m}\left(c_{2}\right)_{n}} \frac{x^{m}}{m !} \frac{y^{n}}{n !} \frac{z^{r}}{r !} \sum_{p=0}^{r} \frac{(-r)_{p}\left(c_{3}\right)_{p}}{\left(2 c_{3}+j\right)_{p}} \frac{2^{p}}{p !} .
$$

Using the definition of ${ }_{p} F_{q}$ in (1.5) for the inner series, we obtain

$$
S=\sum_{m, n, r=0}^{\infty} \frac{(a)_{2 m+n}(b)_{n+r}(-2)^{r}}{\left(c_{1}\right)_{m}\left(c_{2}\right)_{n}} \frac{x^{m}}{m !} \frac{y^{n}}{n !} \frac{z^{r}}{r !}{ }_{2} F_{1}\left[\begin{array}{c}
-r, c_{3} ; \\
2 c_{3}+j ;
\end{array}\right] .
$$


Separating $r$ into even and odd integers and making use of the following identities:

$$
(\alpha)_{2 r}=2^{2 r}(\alpha)_{r}\left(\frac{\alpha}{2}\right)_{r} \quad \text { and } \quad(\alpha)_{r+1}=\alpha(\alpha+1)_{r},
$$

after a little simplification, we have

$$
\begin{aligned}
& S=\sum_{m, n, r=0}^{\infty} \frac{(a)_{2 m+n}(b)_{n+2 r}}{\left(c_{1}\right)_{m}\left(c_{2}\right)_{n}\left(\frac{1}{2}\right)_{r}} \frac{x^{m}}{m !} \frac{y^{n}}{n !} \frac{z^{2 r}}{r !}{ }_{2} F_{1}\left[\begin{array}{l}
-2 r, c_{3} ; \\
2 c_{3}+j ;
\end{array}\right] \\
& -b z \sum_{m, n, r=0}^{\infty} \frac{(a)_{2 m+n}(b+1)_{n+2 r}}{\left(c_{1}\right)_{m}\left(c_{2}\right)_{n}\left(\frac{3}{2}\right)_{r}} \frac{x^{m}}{m !} \frac{y^{n}}{n !} \frac{z^{2 r}}{r !}{ }_{2} F_{1}\left[\begin{array}{r}
-2 r-1, c_{3} ; \\
2 c_{3}+j ;
\end{array}\right] .
\end{aligned}
$$

Finally, using the known results (1.8) and (1.9), after a little simplification, we easily arrive at the right-hand side of (2.1). This completes the proof of (2.1).

\section{Special Cases}

In our main formula (2.1), if we take $j=0, \pm 1$ and \pm 2 , after a little simplification, and we interpret the respective resulting right-hand side with the definition of the triple hypergeometric series $X_{12}$ given in (1.1), we get the following very interesting results:

The case $j=0$.

$$
\begin{gathered}
(1+2 z)^{-b} X_{17}\left(a, b, c_{3} ; c_{1}, c_{2}, 2 c_{3} ; x, \frac{y}{1+2 z}, \frac{4 z}{1+2 z}\right) \\
=X_{12}\left(a, b ; c_{1}, c_{2}, c_{3}+\frac{1}{2} ; x, y, z^{2}\right) .
\end{gathered}
$$

The case $j=1$.

$$
\begin{aligned}
(1+2 z)^{-b} & X_{17}\left(a, b, c_{3} ; c_{1}, c_{2}, 2 c_{3}+1 ; x, \frac{y}{1+2 z}, \frac{4 z}{1+2 z}\right) \\
= & X_{12}\left(a, b ; c_{1}, c_{2}, c_{3}+\frac{1}{2} ; x, y, z^{2}\right) \\
& -\frac{2 b z}{2 c_{3}+1} X_{12}\left(a, b+1 ; c_{1}, c_{2}, c_{3}+\frac{3}{2} ; x, y, z^{2}\right) .
\end{aligned}
$$

The case $j=-1$.

$$
\begin{aligned}
(1+2 z)^{-b} & X_{17}\left(a, b, c_{3} ; c_{1}, c_{2}, 2 c_{3}-1 ; x, \frac{y}{1+2 z}, \frac{4 z}{1+2 z}\right) \\
= & X_{12}\left(a, b ; c_{1}, c_{2}, c_{3}-\frac{1}{2} ; x, y, z^{2}\right) \\
& +\frac{2 b z}{2 c_{3}-1} X_{12}\left(a, b+1 ; c_{1}, c_{2}, c_{3}+\frac{1}{2} ; x, y, z^{2}\right) .
\end{aligned}
$$


The case $j=2$.

$$
\begin{aligned}
(1+2 z)^{-b} & X_{17}\left(a, b, c_{3} ; c_{1}, c_{2}, 2 c_{3}+2 ; x, \frac{y}{1+2 z}, \frac{4 z}{1+2 z}\right) \\
= & X_{12}\left(a, b ; c_{1}, c_{2}, c_{3}+\frac{3}{2} ; x, y, z^{2}\right) \\
& -\frac{2 b z}{c_{3}+1} X_{12}\left(a, b+1 ; c_{1}, c_{2}, c_{3}+\frac{3}{2} ; x, y, z^{2}\right) \\
& +\frac{4 b(b+1) z^{2}}{\left(c_{3}+1\right)\left(2 c_{3}+3\right)} X_{12}\left(a, b+2 ; c_{1}, c_{2}, c_{3}+\frac{5}{2} ; x, y, z^{2}\right) .
\end{aligned}
$$

The case $j=-2$.

$$
\begin{aligned}
(1+2 z)^{-b} & X_{17}\left(a, b, c_{3} ; c_{1}, c_{2}, 2 c_{3}-2 ; x, \frac{y}{1+2 z}, \frac{4 z}{1+2 z}\right) \\
= & X_{12}\left(a, b ; c_{1}, c_{2}, c_{3}-\frac{1}{2} ; x, y, z^{2}\right) \\
& +\frac{2 b z}{c_{3}-1} X_{12}\left(a, b+1 ; c_{1}, c_{2}, c_{3}-\frac{1}{2} ; x, y, z^{2}\right) \\
& +\frac{4 b(b+1) z^{2}}{\left(c_{3}-1\right)\left(2 c_{3}-1\right)} X_{12}\left(a, b+2 ; c_{1}, c_{2}, c_{3}+\frac{1}{2} ; x, y, z^{2}\right)
\end{aligned}
$$

Remark. Clearly Equation (3.1) is the Exton's result given in Equation (1.7) and the Equations (3.2) to (3.5) are closely related to it. The other special cases of (2.1) can also be expressed in terms of $X_{12}$ in the similar manner.

Acknowledgements. This paper was supported by Basic Science Research Program through the National Research Foundation of Korea funded by the Ministry of Education, Science and Technology (2010-0011005).

\section{References}

[1] J. Choi, Notes on formal manipulations of double series, Commun. Korean Math. Soc., 18(4)(2003), 781-789.

[2] J. Choi, A. Hasanov and M. Turaev, Decomposition formulas and integral representations for some Exton typergeometric functions, J. Chungcheong Math. Soc., 24(4)(2011), 745-758.

[3] J. Choi, A. Hasanov and M. Turaev, Linearly independent solutions for the hypergeometric Exton functions $X_{1}$ and $X_{2}$, Honam Math. J., 32(2)(2010), 223-229.

[4] J. Choi, A. Hasanov and M. Turaev, Certain integral representations of Euler type for the Exton function $X_{5}$, Honam Math. J., 32(3)(2010), 389-397. 
[5] J. Choi, A. Hasanov and M. Turaev, Certain integral representations of Euler type for the Exton function $X_{2}$, J. Korean Soc. Math. Edu. Ser. B: Pure Appl. Math., 17(4)(2010), 347-354.

[6] H. Exton, Hypergeometric functions of three variables, J. Indian Acad. Math., 4(1982), $113-119$.

[7] Y. S. Kim, M. A. Rakha and A. K. Rathie, Generalization of Kummer's second summation theorem with applications, Comput. Math. Math. Phys., 50(3)(2010), 387-402.

[8] Y. S. Kim, J. Choi and A. K. Rathie, Remark on two results by Padmanabham for Exton's triple triple hypergeometric series $X_{8}$, Honam Math. J., 27(4)(2005), 603608.

[9] Y. S. Kim and A. K. Rathie, On an extension formulas for the triple hypergeometric $X_{8}$ due to Exton, Bull. Korean Math. Soc., 44(4)(2007), 743-751.

[10] Y. S. Kim, A. K. Rathie and J. Choi, Another method for Padmanabham's transformation formula for Exton's triple triple hypergeometric series $X_{8}$, Commun. Korean Math. Soc., 24(4)(2009), 517-521.

[11] S. W. Lee and Y. S. Kim, An extension of the triple hypergeometric series by Exton, Honam Math. J., 31(1)(2009), 61-71.

[12] E. D. Rainville, Special Functions, Macmillan Company, New York, 1960; Reprinted by Chelsea Publishing Company, Bronx, New York, 1971.

[13] H. M. Srivastava and P. W. Karlsson, Multiple Gaussian Hypergeometric Series, Halsted Press (Ellis Horwood Limited, Chichester), John Wiley and Sons, New York, Chichester, Brisbane and Toronto, 1985. 